\title{
Tracing evolution of spatio-temporal dynamics of the cerebral cortex: cortico-cortical communication dynamics
}

\author{
Per E. Roland ${ }^{1 *}$, Claus C. Hilgetag ${ }^{2,3}$ and Gustavo Deco ${ }^{4}$ \\ 1 Department of Neuroscience and Pharmacology, Faculty of Health Sciences, University of Copenhagen, Copenhagen, Denmark \\ ${ }^{2}$ Department of Computational Neuroscience, University Medical Center Hamburg-Eppendorf, Hamburg, Germany \\ ${ }^{3}$ Department of Health Sciences, Boston University, Boston, MA, USA \\ ${ }^{4}$ Computational Neuroscience Group, Department of Technology, University of Pompeu Fabra, Barcelona, Spain \\ *Correspondence: perrol@sund.ku.dk
}

Edited and reviewed by:

Maria V. Sanchez-Vives, ICREA-IDIBAPS, Spain

Keywords: action potential transmission, connectivity models, multi-area voltage sensitive dye recordings, EEG, MEG

A considerable number of axons from neurons in one cortical area end up on other cortical areas. When one neuron in one cortical area sends an action potential to target neurons in other cortical areas, this is a realization of a cortico-cortical communication. Sensory perception, thinking, and planning of a specific behavior, all rely on the evolution of cortico-cortical communications. The action potentials change the membrane potentials in the target neurons and, in turn, may excite these neurons to produce action potentials and complex patterns of excitation and inhibition in their targets. We launched the special research topic of cortico-cortical communication dynamics to invite contributions that would cast light on such evolution of spatiotemporal action potential and membrane potential dynamics in the cerebral cortex.

The contributions were theoretical models, human EEG, and MEG data and data-driven models, and in vivo experimental data from animals accounting for specific aspects of cortico-cortical communication dynamics.

In a recent in vitro experiment, Branco et al. (2010) show that single dendrites of pyramidal layer 2-3 neurons depolarize more and have larger $\mathrm{Ca}^{2+}$ influx when their depolarization progresses toward the soma, than when depolarization progresses away from the soma. Kiebel and Friston (2011) construct a (developmental) model of the pruning of single synapses and show that they can reproduce the findings of Branco et al. (2010) if the selforganizing pruning follows a Bayesian and information theory derived principle of minimization of free energy. Cortico-cortical communication dynamics can only be comprehensively studied in vivo. In vivo, the neurons and their dendrites are in a high conductance state (Destexhe et al., 2003), and the propagation of depolarizations to the soma and action potential generation may thus be difficult to predict (Williams and Mitchell, 2008). This does not exclude, however, that the model of Kiebel and Friston (2011) may be appropriate in early development and in the formation of cortio-cortical synapses. The pruning of synapses under development and hence the formation of the adult cortical network is the theme of the contribution of van den Bergh et al. (2012). Their model departs from a random network. This network is subsequently shaped by spontaneous ongoing spike activity. After a while the random structure disappears and many small-world sub-networks emerge. As van den Bergh et al. (2012) show, this only happens if the connectivity in the network is larger than a critical value. This is interesting as the developing brain has many cortico-cortical connections that disappear at later stages.

As pointed out in a critical review of cortico-cortical communication dynamics, there are many obstacles precluding the tracing the ms by ms evolution of the spatio-temporal dynamics of the cortex (Roland et al., 2014). Therefore examination of the spatiotemporal dynamics in biologically plausible computational models of neurons may be one way to develop experimentally testable hypotheses. Li and Zhou (2011) made a computational model of neurons in two inter-connected cortical areas. The duration of the delays in communication and the distribution of inhibition in the local network determined whether the neurons would spike in phase or in anti-phase and whether interactions between slow and fast membrane oscillations would produce anti-phase spiking. These findings are pertinent for the hypothesis on cortico-cortical communication through coherence (Fries, 2009).

Facing the obstacles of tracing the spatio-temporal dynamics of cortico-cortical communications at the cellular scale, many scientists choose to study membrane electrical activity at the scale of large neuron populations, and from EEG and MEG signals try to infer putative routes of communication. Banerjee et al. (2012) discuss these methods and point out that there is no consensus as to what constitutes a large-scale network. Further, they show how MEG measurements may be interpreted by combining the empirical analysis with large-scale models of biologically realistic membrane activity. This is what is done in the contributions by Misic et al. (2011) and Vakorin et al. (2011). Their results show that time delays and the number of connections between sources, of MEG signals or EEG signals, contribute to the relation between variance in the signals and information transfer between the sources (Misic et al., 2011; Vakorin et al., 2011).

At the mesoscopic scale one can observe changes in the membrane potentials with voltage sensitive dyes, local field potentials and combine this with recordings of action potentials from a few neurons or single neurons in experimental animals. Harvey and Roland (2013) demonstrate both forward spatiotemporal population membrane dynamics in higher visual areas that after $50 \mathrm{~ms}$ was followed by backward propagation of net-excitation from these areas in experiments with objects moving in the visual 
field. Vinnik et al. (2012) examined the communications from the auditory cortex to the hippocampus and show that the access to fire hippocampal neurons is state dependent. Sleep favors fast reactions of the hippocampal neurons to the extent as only seen for novel sounds in awake animals (Vinnik et al., 2012). Civillico and Contreras (2012) examined how the communication from the thalamus to the barrel cortex is affected by the state of the neurons in the barrel cortex. When the cortical neurons were in an up-state, the local field potentials, the membrane potential increases, and the multiunit activity evoked by a whisker stimulus was smaller than when the whisker stimulus was given just during the early transition from a down-state to an up-state (Civillico and Contreras, 2012).

If one wants to understand how the cerebral cortex works one must be able to trace the evolution of the spatio-temporal transmission of action potentials and membrane conductances down to the cellular scale. As the critical review concludes, this is not possible yet. Assume that a full connectome of the mouse cerebral cortex exists (Bohland et al., 2009). This might help in finding the target neurons in other areas for a given neuron. However, it still remains to identify that source neuron spiking in an experiment and measure the membrane potential changes induced by that neuron on each of the target neurons, as each target neuron may have 1000 other source neurons. One may argue that if this multidimensional cellular dynamics should have any impact on perception and behavior, the dynamics of action potentials and membrane potential dynamics at more coarse scales should organize to make such impacts. The contributions to this special issue are fine examples of the many contemporary attempts to advance theoretical knowledge of cortico-cortical communication dynamics, provide testable hypotheses in this field, and test these hypotheses at the microscopic, mesoscopic, and macroscopic scales.

\section{REFERENCES}

Banerjee, A., Pillai, A. S., and Horwitz, B. (2012). Using large scale neural models to interpret connectivity measures of cortico-cortical dynamics at millisecond temporal resolution. Front. Syst. Neurosci. 5:102. doi: 10.3389/fnsys.2011.00102

Bohland, J. W., Wu, C., Barbas, H., Bokil, H., Bota, M., Breiter, H. C. et al. (2009). A proposal for a coordinated effort for the determination of brainwide neuroanatomical connectivity in model organisms at a mesoscopic scale. PLoS Comput. Biol. 5:e1000334. doi: 10.1371/journal.pcbi.1000334

Branco, T., Clark, B. A., and Häusser, M. (2010). Dendritic discrimination of temporal input sequences i cortical neurons. Science 329, 1671-1675. doi: 10.1126/ science. 1189664
Civillico, E. F., and Contreras, D. (2012). Spatiotemporal properties of sensory responses in vivo are strongly dependent on network context. Front. Syst. Neurosci. 6:25. doi: 10.3389/fnsys.2012.00025

Destexhe, A., Rudolph, M., and Pare, D. (2003). The high-conductance state of neocortical neurons in vivo. Nat. Neurosci. 4, 739-751. doi: 10.1038/nrn1198

Fries, P. (2009). Neuronal gamma-band synchronization as a fundamental process in cortical computation. Annu. Rev. Neurosci. 32, 209-224. doi: 10.1146/annurev.neuro.051508.135603

Harvey, M. A., and Roland, P. E. (2013). Laminar firing and membrane dynamics in four visual areas exposed to two objects moving to occlusion. Front. Syst. Neurosci. 7:23. doi: 10.3389/fnsys.2013.00023

Kiebel, S. J., and Friston, K. J. (2011). Free energy and dendritic self-organization. Front. Syst. Neurosci. 5:80. doi: 10.3389/fnsys.2011.00080

Li, D., and Zhou, C. (2011). Organization of anti-phase synchronization pattern in neural networks: what are the key factors? Front. Syst. Neurosci. 5:100. doi: 10.3389/fnsys.2011.00100

Misic, B., Vakorin, V. A., Paus, T., and McIntosh, A. R. (2011). Functional embedding predicts the variability of neural activity. Front. Syst. Neurosci. 5:90. doi: 10.3389/fnsys.2011.00090

Roland, P. E., Hilgetag, C. C., and Deco, G. (2014). Cortico-cortical communication dynamics. Front. Syst. Neurosci. 8:19. doi: 10.3389/fnsys.2014. 00019

Vakorin, V. A., Misic, B., Krakovska, O., and McIntosh, A. R. (2011). Empirical and theoretical aspects of generation and transfer of information in a neuromagnetic source network. Front. Syst. Neurosci. 5:96. doi: 10.3389/fnsys.2011. 00096

van den Bergh, D., Gong, P., Breakspear, M., and van Leuwen, C. (2012). Fragmentation: loss of global coherence or breakdown of modularity in functional architecture? Front. Syst. Neurosci. 6:20. doi: 10.3389/fnsys.2012. 00020

Vinnik, E., Antopolsky, S., Itskov, P. M., and Diamond, M. E. (2012). Auditory stimuli elicit hippocampal neuronal responses during sleep. Front. Syst. Neurosci. 6:49. doi: 10.3389/fnsys.2012.00049

Williams, S. R., and Mitchell, S. J. (2008). Direct measurement of somatic voltage clamp errors in central neurons. Nat. Neurosci. 11, 790-798. doi: $10.1038 / \mathrm{nn} .2137$

Conflict of Interest Statement: The authors declare that the research was conducted in the absence of any commercial or financial relationships that could be construed as a potential conflict of interest.

Received: 25 September 2013; accepted: 15 April 2014; published online: 05 May 2014. Citation: Roland PE, Hilgetag CC and Deco G (2014) Tracing evolution of spatiotemporal dynamics of the cerebral cortex: cortico-cortical communication dynamics. Front. Syst. Neurosci. 8:76. doi: 10.3389/fnsys.2014.00076

This article was submitted to the journal Frontiers in Systems Neuroscience.

Copyright (C) 2014 Roland, Hilgetag and Deco. This is an open-access article distributed under the terms of the Creative Commons Attribution License (CC BY). The use, distribution or reproduction in other forums is permitted, provided the original author(s) or licensor are credited and that the original publication in this journal is cited, in accordance with accepted academic practice. No use, distribution or reproduction is permitted which does not comply with these terms. 